\title{
Barriers physicians face when referring patients to cardiac rehabilitation: a narrative review
}

\author{
Carol Elsakr ${ }^{1}$, David A. Bulger ${ }^{2}$, Sherif Roman ${ }^{3}$, Irene Kirolos ${ }^{4}$, Rami N. Khouzam ${ }^{4}$ \\ ${ }^{1}$ Department of Medicine, The George Washington University School of Medicine \& Health Sciences, Washington, DC, USA; ${ }^{2}$ Department \\ of Internal Medicine, University of Tennessee Health Science Center, Memphis, TN, USA; ${ }^{3}$ Department of Medicine, Cairo University, Giza \\ Governorate, Egypt; ${ }^{4}$ Department of Internal Medicine, Division of Cardiovascular Diseases, University of Tennessee Health Science Center, \\ Memphis, TN, USA \\ Contributions: (I) Conception and design: C Elsakr, RN Khouzam; (II) Administrative support: RN Khouzam; (III) Provision of study materials or \\ patients: None; (IV) Collection and assembly of data: C Elsakr; (V) Data analysis and interpretation: C Elsakr; (VI) Manuscript writing: All authors; \\ (VII) Final approval of manuscript: All authors. \\ Correspondence to: Carol Elsakr, BS. Department of Medicine, The George Washington University School of Medicine \& Health Sciences, 2300 I St \\ NW, Washington, DC 20052, USA. Email: celsakr@gwu.edu.
}

\begin{abstract}
While cardiac rehabilitation (CR) has been shown to be a beneficial form of secondary prevention for patients with cardiovascular disease, barriers of referral to CR still exist for patients. Barriers that specifically make it difficult for physicians to make the referral could be worthwhile to examine. This narrative review hypothesizes that increasing awareness and education on the various aspects of CR as well as simplifying the referral process could lead to increased referral rates as they target physician-related barriers. This narrative review seeks to further understand the physician-related barriers of low CR awareness and hindering referral processes. A search in Scopus was conducted with preference for articles examining CR referral strategies used by physicians; physicians' awareness of CR programs; physicians' perceptions, beliefs, or knowledge of the benefits of CR; or physicians' experience with or understanding of the selection process of CR programs, including indications for referral. Two systematic reviews and two observational studies were selected for discussion. Three of the selected studies had findings supporting the notion that increasing physicians' awareness of CR could impact referral rates. One of the studies evaluated the perceptions that physicians and CR programs had on various referral strategies. While more study is needed to assess the actual level of knowledge and awareness physicians have regarding CR, this review supports using educational interventions as well as targeting various aspects of the referral process for improving referral rates.
\end{abstract}

Keywords: Cardiac rehabilitation (CR); clinical competence; consultation; education; physicians; referral

Submitted Jun 30, 2019. Accepted for publication Jul 12, 2019.

doi: $10.21037 /$ atm.2019.07.61

View this article at: http://dx.doi.org/10.21037/atm.2019.07.61

\section{Introduction}

Cardiac rehabilitation (CR) is a multidisciplinary program that aims to help patients recover from a cardiac event and lower their risk of death via lifestyle interventions (1). While CR has been shown to have mortality benefits in cardiovascular patients (2-7), many patients still lack access to these services partially due to lack of referrals, as commented on by Abreu (8). This is especially true among female patients (9-14), non-White patients $(15,16)$, and patients without insurance coverage (17-19).

Focusing particularly on physician-related barriers to CR referrals could be useful (20-22). Three potential ways to assist physicians in referring patients to $\mathrm{CR}$ are to reduce the effort required by simplifying the referral process, increase awareness of CR programs, and clarify the benefits of and selection for patient candidates by CR programs (23). An overly-complex referral process creates a barrier in 
the form of increased time and effort required to refer patients (24). Education on the benefits of and selection process for these programs should provide physicians the tools needed to perform these referrals (24-26). This narrative review attempts to better understand barriers relevant to physicians and how to address them.

\section{Methods}

A series of Scopus searches were performed to find relevant articles addressing the barriers in CR referrals. The keywords "cardiac rehab" and either "refer*", "order*", or "prescri*" were used. A portion of the search results was screened based on title and/or abstract for possible relevance to the topic of physician-related barriers to CR referral. References within those articles as well as related articles were also screened for inclusion.

The articles were further narrowed down by picking articles that examined, discussed, or had results related to CR referral of patients as well as related to either (I) CR referral strategies used by physicians, (II) physicians' awareness of CR programs, (III) physicians' perceptions, beliefs, or knowledge of the benefits of CR, or (IV) physicians' experience with or understanding of the selection process of CR programs, including indications for referral.

Preference was given to articles published between January 2000 and July 2019. Articles were also preferred if they were randomized controlled trials, observational studies, or systematic reviews rather than book sections, conference proceedings, guidelines, commentaries, editorials, expert opinions, case reports, case series, narrative reviews, or scoping reviews. Articles whose results were examined in included systematic reviews were excluded in this review in order to reduce redundancy.

\section{Results}

Ultimately, four articles were selected for review. These four articles included two systematic reviews $(27,28)$ and two observational studies $(29,30)$.

Ghisi et al. reviewed 10 observational studies primarily from the United States and Canada that used CR referral as an outcome $(17,23,31-38)$ and 4 that had physicians as participants $(21,23,34,38)$. Two of their reviewed studies $(33,34)$ found a correlation between specialty and CR referral with primary care associated with less referrals than specialty services, such as cardiology or cardiac surgery (28).
Clark et al.'s review had a total of 880 patients, 69 nonmedical caregivers, and 163 medical professionals (27). They found that while physicians were seen as not fully aware of the benefits, nature, and indications of CR $(26,39,40)$.

Moradi et al. conducted a cross-sectional survey of 122 cardiologists from 11 major training centers in Iran asking questions on the nature of CR, its effect on mortality, and factors affecting CR rates (30). Overall, while $47.9 \%$ of respondents reported having CR centers available to them, only $6.6 \%$ had regular CR education. Furthermore, $90.7 \%$ of respondents reported a CR referral rate of less than $15 \%$, which most attributed to lack of awareness of the benefits and nature of CR programs (30). Grace et al. sent out a cross-sectional anonymous survey in Canada, which received responses from $92 \mathrm{CR}$ programs and 71 cardiac specialists (29). The survey asked cardiac specialists and CR programs to report the CR referral strategies they used and to rate various types of referral strategies in terms of effectiveness (29). Cardiac specialists' top four rated CR strategies (score of 4.4/5) were having CR referrals included on discharge order-sets, which was used by $67.6 \%$ of cardiac specialist respondents and $61.4 \%$ of CR program respondents; CR discussion with the patient by an allied health care provider (4.4/5) both at bedside or over the phone following discharge, which was used by $63.4 \%$ of cardiac specialist respondents and 59\% of CR program respondents; incorporating CR referrals into a standard post-discharge follow-up clinic visit; and setting up CR intake appointments for patients prior to discharge. CR programs' top three rated CR strategies (4.1/5) were setting up CR intake appointments for patients prior to discharge, having the Hospital Medical Advisory Committee CR approve CR referral as a standard order, and including CR referrals in electronic hospital records (29). Grace et al. also found that CR programs were experiencing space constraints (33.8\% of CR program respondents), staff fatigue (18.2\%), and longer waiting lists (31.2\%) after implementing referral strategies.

\section{Discussion}

\section{Lack of CR awareness}

Clark et al. and Moradi et al. identified physician awareness of the benefits, selection process, and nature of CR programs as potential factors in CR referral $(27,30)$. While Ghisi et al. found evidence for primary care physicians being less 
likely to refer patients to cardiac rehabilitation than cardiac surgeons or cardiologists $(28,33,34)$, Moradi et al. found in Iran that both cardiologists and cardiologist fellows had low CR referral rates (30). This is interesting as one might assume that primary care physicians may be referring less out of lack of CR exposure via cardiology training, yet the results of Moradi et al. question this (30). One study reviewed in Ghisi et al. (28) sought to determine the reasons for why primary care physicians refer less than cardiac specialists; the findings were that in Ontario, primary care physicians were more likely to report issues with the referral process (e.g., lack of standardized referral forms, lack of discharge communication from cardiac rehabilitation, inconvenience) as potentially affecting their referral rate than cardiac specialists (who, in turn, were more likely to endorse a culture that promotes cardiac rehabilitation referral) (23). Many of the primary care physician respondents to a qualitative survey reported that geographic accessibility (43.8\% of respondents) and patient motivation (21.3\%) were the most important factors affecting referral to cardiac rehabilitation rather than patient benefit (11.2\%), medical characteristics of patients (6.7\%), or other reasons (16.7\%), which included issues with the referral process (23). Many of the cardiac specialist respondents also reported that patient motivation (23.8\% of respondents) was one of the most important factors affecting cardiac rehabilitation; however, cardiac specialists did endorse patient benefit (15.9\%) and medical characteristics of patients $(11.1 \%)$ being important factors as well, with less respondents than primary care physicians endorsing other reasons, such as the referral process itself, being a factor (12.4\%) (23). Based on these findings, it seems that primary care physicians' lower CR referral rates could be due to lack of awareness of the CR referral process but also could be due to patient-specific factors.

Clark et al. found lack of knowledge to be a potential barrier to CR referral (27). Their systematic review found that physicians appeared to be lacking in knowledge regarding what cardiac rehabilitation entails, the value of cardiac rehabilitation, and when patients should be referred to it from the perspectives of patients and healthcare professionals $(26,27,39,40)$. On the other hand, Grace et al. did find that cardiac specialists on average gave themselves a high score of 4.4 out of 5 in terms of how extensive they believe their awareness of cardiac rehabilitation's benefits is (29). One possible explanation includes the timing of the study, which was likely done later than the other discussed observational studies $(23,30)$ as well as the studies included in the systematic reviews of this paper.
To summarize, lack of CR awareness among physicians has been identified as a potential barrier to CR referral for both cardiac and non-cardiac specialists $(27,30)$. However, the results of Grace et al. do question whether this barrier still exists or not in North America, and more studies assessing lack of knowledge that rely on non-physician self-reports as well as use other methodology besides selfreports could help determine this (29). This barrier could be addressed via workshops or cardiac health organization meetings $(27,30)$.

\section{Referral strategies}

Grace et al. discusses how various strategies could be used to increase CR referral rates (29). Interestingly, the most commonly used referral strategies by both cardiac specialists and CR programs were CR discharge order-sets, bedside or over the phone post-discharge discussions about $\mathrm{CR}$ with an allied health professional, and CR referral form completion by an allied health professional. The cardiac specialists also found incorporating CR into the standard post-discharge follow up clinic visit and making a CR intake appointment prior to discharge helpful. CR programs additionally highly rated having the Hospital Medical Advisory Committee approve CR referrals as standard orders for all eligible patients as well as an electronic medical record process. While there were significant differences in ratings between cardiac specialists and CR programs with many of these strategies, they both rated peer mentor CR discussion as a less effective strategy.

Ghisi et al. discussed how the study done by Grace $e t$ al. found that referral process issues could partially explain the differences in referral rate between primary care physicians and cardiac specialists, such as the lack of standardized referral forms $(23,28)$. Other issues with the referral process were reviewed in Clark et al., such as the lack of reimbursement for physicians who do refer as well as the lack of time to refer $(26,27,39,40)$.

Clark et al. also discussed Grace et al., which highlighted the role of communication between healthcare providers, patients, and CR programs (e.g., communication by mail and staff follow up with patients after order has been made); territoriality (e.g., fear of losing patients to CR programs); and automatic referrals $(27,41)$. Clark et al. also reviewed Gurewich et al., another study that was initially included in this paper for discussion and that used semi-structured interviews with 16 directors of cardiac rehabilitation programs and 6 presidents of cardiac 
rehabilitation state associations in states that either had high or low rates of cardiac rehabilitation use (based on 1997 reports for Medicare beneficiaries above the age 65) $(27,42)$. The relevant results of this qualitative study were that amongst states of high cardiac rehabilitation use, cardiac rehabilitation programs were more likely to actively seek out referrals by advising physicians to issue a referral, rely on various automated systems (e.g., electronic patient records, standard orders, and other systematic, non manual methods for identifying eligible patients and prompting staff to meet with the patient and/or follow-up with their referring physician), and to have staff on the lookout for eligible patients or take responsibility for determining which patients are eligible for cardiac rehabilitation than programs in states of low cardiac rehabilitation use.

To summarize, targeting the referral process itself could potentially be effective in improving referral rates, and the studies discussed above provide many possible solutions that should be attempted in future studies.

\section{Limitations}

This narrative review was not a comprehensive one as its goal was to review a portion of relevant articles related to this subject. There were articles that were possibly relevant to this topic and may have even met the authors' preferences that were excluded.

Limitations of this review article include location bias (only the Scopus database was searched), language bias, and citation bias. As this was a narrative review, eligibility criteria and screening were not as stringent as those of other reviews. No official data extraction table was created, which could lead to potential reporting bias in this review. Finally, no official tools for quality assessment of articles or for results synthesis were used in the writing of this review.

Limitations existed for the selected articles above as well (27-30). The systematic reviews for the most part examined cross-sectional studies and qualitative studies, rather than studies where causality can be deduced $(27,28)$. Neither of them performed a meta-analysis, with one of them stating it was not done due to too many sources of heterogeneity across studies (28).

\section{Potential interventions and future directions}

Some authors have commented that improving referral rates to CR does not mean that enrollment or participation rates will also improve $(43,44)$. The gap between referral rate and the following enrollment rate could be explained by a number of factors $(45,46)$, such as if healthcare providers expressed less optimism and reassurance during discussions about CR (47). Nevertheless, intervening at the level of referral is still key for improving enrollment rates (48).

Studies have also discussed different forms of automated referrals, comprehensive inpatient discharge order sets, and eReferral strategies as possible solutions for optimizing the referral process $(27,28,44)$. Moradi et al. and Grace et al. discussed linking online directories of CR programs with referral forms $(23,30)$. Grace et al. also recommended the use of standardized referral forms based on responses from primary care physicians (23). Another study even discussed the use of a motivational, educational CR letter to patients as prompts for physicians to start the referral process (28). Conversely, Gallagher et al. recommend a "liaison type of referral strategy", as it is more educational to patients, which could lead to increased participation after referral (49). This strategy is supported by a review done by Gravely-Witte et al. showing automatic referrals achieved the highest rates of referral, whereas combining liaison referral and automatic referral achieved the highest rates of enrolment (50).

Lastly, Grace et al. discusses their findings on how cardiac rehabilitation programs' resources have been unexpectedly overwhelmed due to the increase in referrals due to successful strategies, which could be an area of focus for future studies (29).

\section{Conclusions}

In conclusion, while more study is needed on the awareness physicians have of CR, this review overall supports using educational interventions for improving CR referral rates. Furthermore, this review supports targeting various aspects of the referral process itself for improving CR referral rates.

\section{Acknowledgments}

None.

\section{Footnote}

Conflicts of Interest: The authors have no conflicts of interest to declare.

Etbical Statement: The authors are accountable for all aspects of the work in ensuring that questions related to the accuracy or integrity of any part of the work are 
appropriately investigated and resolved.

\section{References}

1. Freeman AM, Taub PR, Lo HC, et al. Intensive Cardiac Rehabilitation: an Underutilized Resource. Curr Cardiol Rep 2019;21:19.

2. Anderson L, Oldridge N, Thompson DR, et al. ExerciseBased Cardiac Rehabilitation for Coronary Heart Disease: Cochrane Systematic Review and Meta-Analysis. J Am Coll Cardiol 2016;67:1-12.

3. Goel K, Lennon RJ, Tilbury RT, et al. Impact of cardiac rehabilitation on mortality and cardiovascular events after percutaneous coronary intervention in the community. Circulation 2011;123:2344-52.

4. Hammill BG, Curtis LH, Schulman KA, et al. Relationship between cardiac rehabilitation and long-term risks of death and myocardial infarction among elderly Medicare beneficiaries. Circulation 2010;121:63-70.

5. Hermann M, Witassek F, Erne P, et al. Impact of cardiac rehabilitation referral on one-year outcome after discharge of patients with acute myocardial infarction. Eur J Prev Cardiol 2019;26:138-44.

6. Norris CM, Jensen LA, Galbraith PD, et al. Referral rate and outcomes of cardiac rehabilitation after cardiac catheterization in a large Canadian city. J Cardiopulm Rehabil 2004;24:392-400.

7. Rauch B, Davos CH, Doherty P, et al. The prognostic effect of cardiac rehabilitation in the era of acute revascularisation and statin therapy: A systematic review and meta-analysis of randomized and non-randomized studies - The Cardiac Rehabilitation Outcome Study (CROS). Eur J Prev Cardiol 2016;23:1914-39.

8. Abreu A. Cardiac rehabilitation after acute myocardial infarction: Still insufficient referral! Int J Cardiol 2018;261:28-9.

9. Ades PA, Waldmann ML, Polk DM, et al. Referral patterns and exercise response in the rehabilitation of female coronary patients aged greater than or equal to 62 years. Am J Cardiol 1992;69:1422-5.

10. Beckstead JW, Pezzo MV, Beckie TM, et al. Physicians' tacit and stated policies for determining patient benefit and referral to cardiac rehabilitation. Med Decis Making 2014;34:63-74.

11. Colella TJ, Gravely S, Marzolini S, et al. Sex bias in referral of women to outpatient cardiac rehabilitation? A metaanalysis. Eur J Prev Cardiol 2015;22:423-41.

12. Cottin Y, Cambou JP, Casillas JM, et al. Specific profile and referral bias of rehabilitated patients after an acute coronary syndrome. J Cardiopulm Rehabil 2004;24:38-44.

13. Jackson L, Leclerc J, Erskine Y, et al. Getting the most out of cardiac rehabilitation: a review of referral and adherence predictors. Heart 2005;91:10-4.

14. Scott LA, Ben-Or K, Allen JK. Why are women missing from outpatient cardiac rehabilitation programs? A review of multilevel factors affecting referral, enrollment, and completion. J Womens Health (Larchmt) 2002;11:773-91.

15. Allen JK, Scott LB, Stewart KJ, et al. Disparities in women's referral to and enrollment in outpatient cardiac rehabilitation. J Gen Intern Med 2004;19:747-53.

16. Beatty AL, Li S, Thomas L, et al. Trends in referral to cardiac rehabilitation after myocardial infarction: data from the National Cardiovascular Data Registry 2007 to 2012. J Am Coll Cardiol 2014;63:2582-3.

17. Bittner V, Sanderson B, Breland J, et al. Referral patterns to a University-based cardiac rehabilitation program. Am J Cardiol 1999;83:252-5, A5.

18. Cortés O, Arthur HM. Determinants of referral to cardiac rehabilitation programs in patients with coronary artery disease: a systematic review. Am Heart J 2006;151:249-56.

19. Missik E. Women and cardiac rehabilitation: accessibility issues and policy recommendations. Rehabil Nurs 2001;26:141-7.

20. Aragam KG, Dai D, Neely ML, et al. Gaps in referral to cardiac rehabilitation of patients undergoing percutaneous coronary intervention in the United States. J Am Coll Cardiol 2015;65:2079-88.

21. Grace SL, Gravely-Witte S, Brual J, et al. Contribution of patient and physician factors to cardiac rehabilitation enrollment: a prospective multilevel study. Eur J Cardiovasc Prev Rehabil 2008;15:548-56.

22. Shanks LC, Moore SM, Zeller RA. Predictors of cardiac rehabilitation initiation. Rehabil Nurs 2007;32:152-7.

23. Grace SL, Grewal K, Stewart DE. Factors affecting cardiac rehabilitation referral by physician specialty. J Cardiopulm Rehabil Prev 2008;28:248-52.

24. Thomas RJ. Cardiac rehabilitation/secondary prevention programs: a raft for the rapids: why have we missed the boat? Circulation 2007;116:1644-6.

25. LaBresh KA, Gliklich R, Liljestrand J, et al. Using â€œGet With The Guidelinesâ€ $€$ to Improve Cardiovascular Secondary Prevention. Jt Comm J Qual Saf 2003;29:539-50.

26. Scott LB, Allen JK. Providers' Perceptions of Factors Affecting Women's Referral to Outpatient Cardiac Rehabilitation Programs: AN EXPLORATORY STUDY. 
J Cardiopulm Rehabil 2004;24:387-91.

27. Clark AM, King-Shier KM, Duncan A, et al. Factors influencing referral to cardiac rehabilitation and secondary prevention programs: a systematic review. Eur J Prev Cardiol 2013;20:692-700.

28. Ghisi GLM, Polyzotis P, Oh P, et al. Physician factors affecting cardiac rehabilitation referral and patient enrollment: a systematic review. Clin Cardiol 2013;36:323-35.

29. Grace SL, Tan Y, Simpson C, et al. Perceptions of cardiac specialists and rehabilitation programs regarding patient access to cardiac rehabilitation and referral strategies. J Cardiopulm Rehabil Prev 2012;32:135-40.

30. Moradi B, Maleki M, Esmaeilzadeh M, et al. Physicianrelated factors affecting cardiac rehabilitation referral. J Tehran Heart Cent 2011;6:187-92.

31. Dunlay SM, Witt BJ, Allison TG, et al. Barriers to participation in cardiac rehabilitation. Am Heart J 2009;158:852-9.

32 Ayanian JZ, Landrum MB, Guadagnoli E, et al. Specialty of ambulatory care physicians and mortality among elderly patients after myocardial infarction. N Engl J Med 2002;347:1678-86.

33. Barber K, Stommel M, Kroll J, et al. Cardiac rehabilitation for community-based patients with myocardial infarction: Factors predicting discharge recommendation and participation. J Clin Epidemiol 2001;54:1025-30.

34. Grace SL, Evindar A, Abramson BL, et al. Physician management preferences for cardiac patients: factors affecting referral to cardiac rehabilitation. Can J Cardiol 2004;20:1101-7.

35. Pell J, Pell A, Morrison C, et al. Retrospective study of influence of deprivation on uptake of cardiac rehabilitation. BMJ 1996;313:267-8.

36. Scott IA, Duke AB, Darwin IC, et al. Variations in indicated care of patients with acute coronary syndromes in Queensland hospitals. Med J Aust 2005;182:325-30.

37. Stiller JJ, Holt MM. Factors influencing referral of cardiac patients for cardiac rehabilitation. Rehabil Nurs 2004;29:18-23.

38. Suter P, Bona S, Suter WN. Views of Arkansas Physicians on Cardiac Rehabilitation. J Cardiopulm Rehabil Prev 1992;12:32-5.

39. Allen M, Mann K, Putnam W, et al. Prescribing Exercise for Cardiac Patients: Knowledge, Practices, and Needs of Family Physicians and Specialists. J Cardiopulm Rehabil
2000;20:333-9.

40. Corrrigan M, Cupples ME, Smith SM, et al. The contribution of qualitative research in designing a complex intervention for secondary prevention of coronary heart disease in two different healthcare systems. BMC Health Serv Res 2006;6:90.

41. Grace SL, Krepostman S, Brooks D, et al. Referral to and discharge from cardiac rehabilitation: key informant views on continuity of care. J Eval Clin Pract 2006;12:155-63.

42. Gurewich D, Prottas J, Bhalotra S, et al. System-level factors and use of cardiac rehabilitation. J Cardiopulm Rehabil Prev 2008;28:380-5.

43. Scott LB. Referral to outpatient cardiac rehabilitation: intervention research at the patient, provider, and health system levels. Nat Clin Pract Cardiovasc Med 2008;5:671-2.

44. Boyden T, Rubenfire M, Franklin B. Will Increasing Referral to Cardiac Rehabilitation Improve Participation? Prev Cardiol 2010;13:192-201.

45. Turk-Adawi KI, Oldridge NB, Tarima SS, et al. Cardiac rehabilitation enrollment among referred patients: patient and organizational factors. J Cardiopulm Rehabil Prev 2014;34:114-22.

46. Weingarten MN, Salz KA, Thomas RJ, et al. Rates of enrollment for men and women referred to outpatient cardiac rehabilitation. J Cardiopulm Rehabil Prev 2011;31:217-22.

47. Pourhabib S, Kentner AC, Grace SL. The impact of patient-healthcare provider discussions on enrollment in cardiovascular rehabilitation. J Rehabil Med 2014;46:924-31.

48. Mitoff PR, Wesolowski M, Abramson BL, et al. Patientprovider communication regarding referral to cardiac rehabilitation. Rehabil Nurs 2005;30:140-6.

49. Gallagher R, Neubeck L, Du H, et al. Facilitating or getting in the way? The effect of clinicians' knowledge, values and beliefs on referral and participation. Eur J Prev Cardiol 2016;23:1141-50.

50. Gravely-Witte S, Leung YW, Nariani R, et al. Effects of cardiac rehabilitation referral strategies on referral and enrollment rates. Nat Rev Cardiol 2010;7:87-96.

Cite this article as: Elsakr C, Bulger DA, Roman S, Kirolos I, Khouzam RN. Barriers physicians face when referring patients to cardiac rehabilitation: a narrative review. Ann Transl Med 2019;7(17):414. doi: 10.21037/atm.2019.07.61 